\title{
Circular-Slide Wave Energy Converter
}

\author{
Shuqin Liu ${ }^{1, a}$, Deguang $\mathrm{Li}^{2, \mathrm{~b}}, \mathrm{H}$. Ming Chen ${ }^{3, \mathrm{c}}$ \\ ${ }^{1,2}$ School of Electrical Engineering, Shandong University, Jinan, Shandong, China \\ ${ }^{3}$ Wave Energy Technologies,Albany, NY, USA

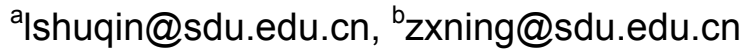 \\ chmchen@verizon.net
}

Keywords: wave energy converter, circular track, sliding mass, artificial spring, random wave

\begin{abstract}
A CS-WEC utilizes an artificial spring created by feedback control of sliding-mass angle on the circular track; it is free of problems associated with mechanical springs. This paper also demonstrates that the harvested power is always more with the spring than without, and with the spring, the power harvest is insensitive to load.
\end{abstract}

\section{Introduction}

For small-to-moderate power applications in the deep ocean where anchoring is impractical, such as charging batteries on weather buoys, a "shaking" type of wave energy converters (WEC) is possible. A WEC of this type is similar to a rechargeable flashlight that is energized by hand shaking. It consists of a generator mass with permanent magnets that hangs from springs [1]. The mass moves vertically in response to wave heaving motion. The natural frequency of the massspring system must be close to the predominant wave frequency so that large vibration amplitudes can be achieved for effective energy harvesting. However, ocean wave frequencies are naturally low, so soft springs are required to achieve low frequencies, which leads to impractically large vertical deflections of the generator mass. Therefore, this conventional WEC is usually long and bulky and the springs represent a design weakness.

Recently proposed was a different concept of shaking WEC with a mass that slides in a circular track under gravity in response to wave-induced buoy pitch/roll, as illustrated in Figure 1 . The conceptual Circular-Slide (CS) WEC includes the following major components: sliding mass, circular sliding track, connecting arm, gearbox, generator, and encoder as shown in Figure 2.

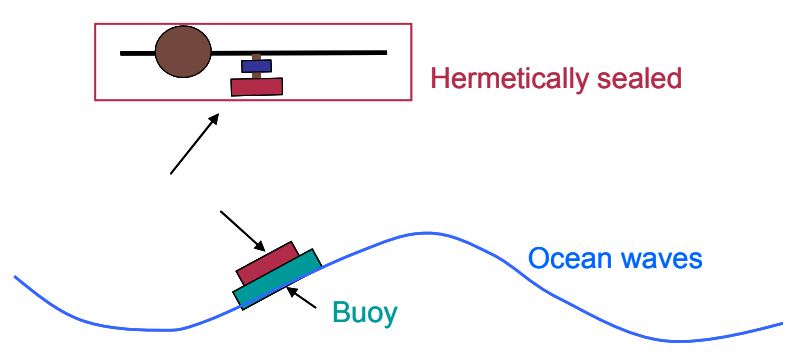

Fig. 1 - CS-WEC excited by pitch/roll of waves

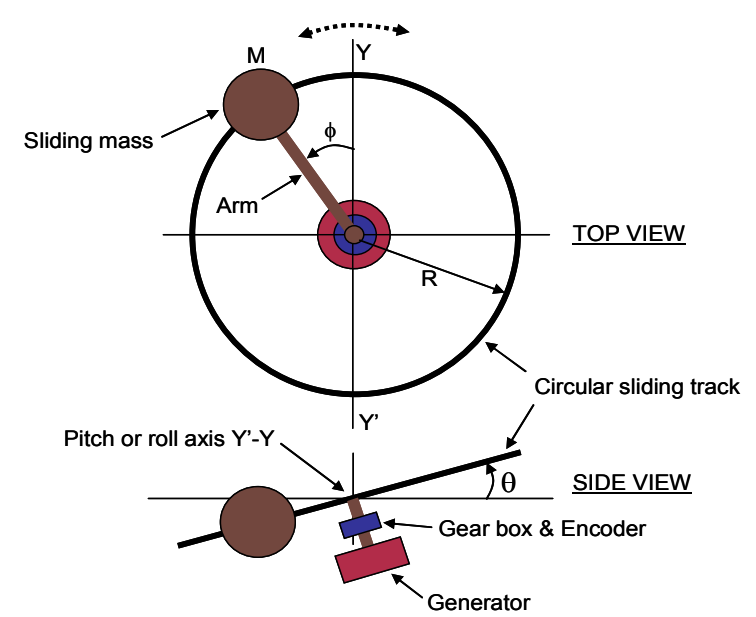

Fig. 2 - major components of CS-WEC

For protection from the harsh ocean environment, all of the components would be mounted inside a hermetically sealed box. 
A key feature of CS-WEC is an artificial spring created by feedback control of the sliding mass location angle on the track. This spring together with the circular track supporting the weight of the sliding mass, eliminates the mechanical spring problems associated with the conventional WEC. Another advantage is that the spring rate is on-line adjustable to make the system resonating with waves and harvesting power efficiently. In this paper, the difference of power harvesting with and without using the artificial spring will be demonstrated.

\section{Description of CS-WEC}

The sliding mass is a weight with a circular or rectangular cross-section that slides on a lowfriction track. The connecting arm is a light structure that connects the sliding mass to the input shaft of a gearbox. The encoder is mounted at the input shaft of the gearbox for measuring the angular displacement of the sliding mass, which is feedback controlled to create an artificial torsional spring using the generator as the actuator or motor. Responding to the pitch/roll motions of waves, this artificial spring would make the sliding mass resonate, or move back and forth on the circular track, at a dominant wave frequency. The angular motion of the sliding mass is amplified by the gearbox to drive the generator and produce electrical power.

Not shown in the Figure 2 is a system controller. It performs functions including:

(1)Use sensors and/or recorded data to estimate the dominant wave period for artificial spring.

(2)Perform feedback control of encoder signal and create reaction torque in generator for the spring function.

(3)Control generator load for maximal output power.

(4)Monitor system parameters and communicate with an outside system.

\section{Dynamics of CS-WEC}

The relevant dynamic parameters and forces associated with the CS-WEC are presented in Figure 3. Neglecting the polar moments of inertia of the connecting arm, generator rotor, etc., the equation of motion of a CS-WEC is:

$$
M R^{2} \frac{d^{2} \phi}{d t^{2}}+B \frac{d \phi}{d t}+K(\phi-\alpha)=\operatorname{Mgsin}(\theta) \cos (\phi-\alpha) R
$$

Where

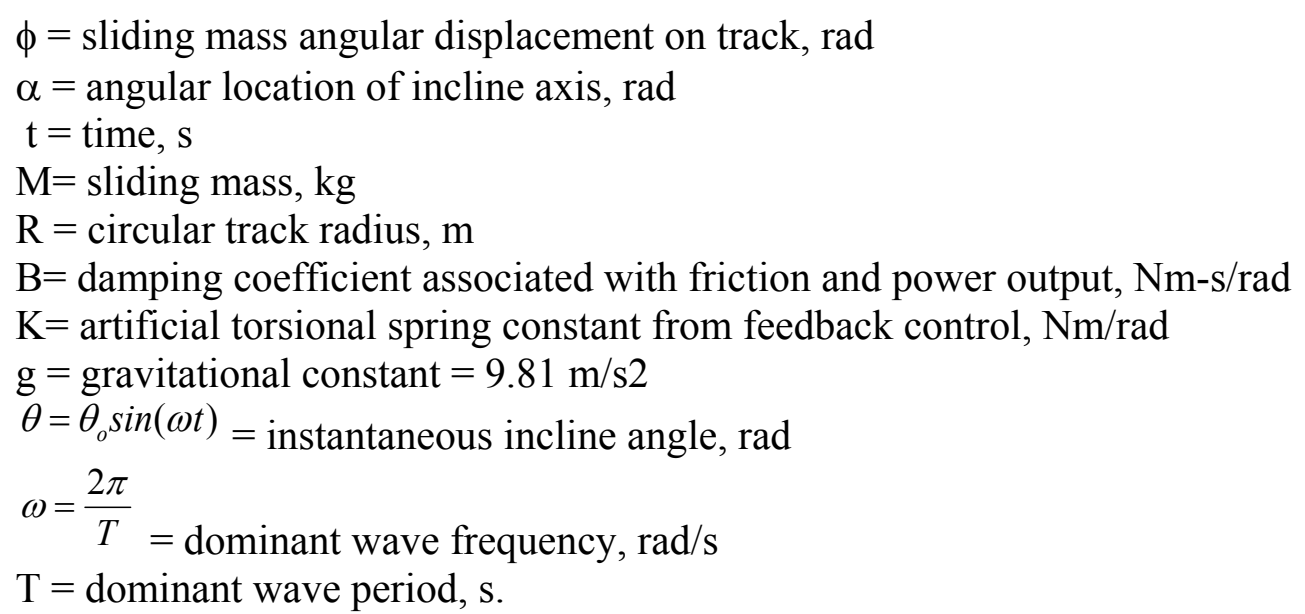

The artificial torsional spring is a proportional feedback control of measured sliding mass angle on the track. The proportional constant, i.e., the torsional stiffness is set to equal to MR $2 \omega 2$. This implies the controlled spring torque is always equal to the inertial torque but opposite in sign, which is a requirement for system resonance. 
To make the artificial spring practical, the motor must be a low power consumption device. The motoring function is implemented in the generator to keep the hardware simple and to reduce weight and volume.

Treating a deep sea ocean swell as a sinusoidal wave, the incline angle amplitude in radian can be derived as:

$$
\theta_{o}=\frac{H}{2} \frac{2 \pi}{\lambda}=\frac{(2 \pi)^{2}}{2 g} \frac{H}{T^{2}} \approx 2 \frac{H}{T^{2}}
$$

Where $\mathrm{H}=$ wave height, $\mathrm{m}$

$$
\lambda=\frac{g T^{2}}{2 \pi}=\text { deep water wave length, } \mathrm{m}
$$

It has been proven $[2,3]$ that the average power $(\mathrm{P})$ at resonance is:

$$
\mathrm{P}=20 \pi \eta \mathrm{MRH} / \mathrm{T} 3
$$

Where $\eta=$ system efficiency, e.g., 0.75 .

The scaling law (3) indicates that the average power of a tuned circular-slider wave energy converter is directly proportional to the sliding mass $(M)$, the circular sliding track radius $(R)$, the significant wave height $(H)$ and inversely proportional to the wave period $(T)$ cubed.

On average, world-wide Ocean has a wave height of about $2 \mathrm{~m}$ and dominant period of about $10 \mathrm{~s}$. For those average conditions, the CS-WEC would produce average power (in watts) in the following table, according to the scaling law (3).

\begin{tabular}{|c|c|c|c|}
\hline & \multicolumn{3}{|c|}{ Radius (m) } \\
\hline Mass (kg) & 0.5 & 1.0 & 2.0 \\
\hline 25 & 1 & 2 & 5 \\
\hline 50 & 2 & 5 & 9 \\
\hline 100 & 5 & 9 & 18 \\
\hline
\end{tabular}

\section{Control Reffrence Search For Artificial Spring}

The dominant axis of a roll/pitch motion changes with time and with orientation of the CS-WEC housing. The on-board controller should be able to track the roll/pitch axis relative to the housing.

On Figure 3, the line " $\phi=0$ " represents a fixed diameter on the CS-WEC housing. The line " $\phi$ $=\alpha$ " represents the axis of the dominant roll/pitch motion, which has an incline angle " $\theta$ " as shown by the "A-A" view of Figure 3.

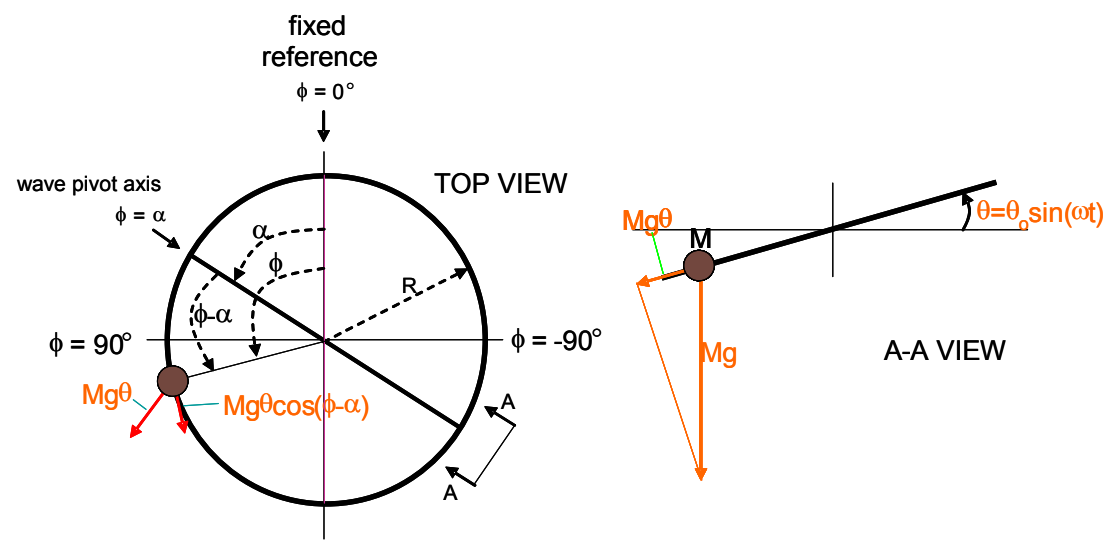

Fig. 3 - Relevant parameters of a circular-slide wave energy converter.

The artificial spring has to set its reference for feedback control of the encoder measurement at " $\phi=\alpha$ " to achieve maximum power. Unless there is a sensor to detect the angle " $\theta$ ", the on-board controller has to search for the " $\alpha$ " value by gradually changing its reference until maximum power is achieved. To prove this search concept dynamically feasible, an example is presented in Figure 4. 


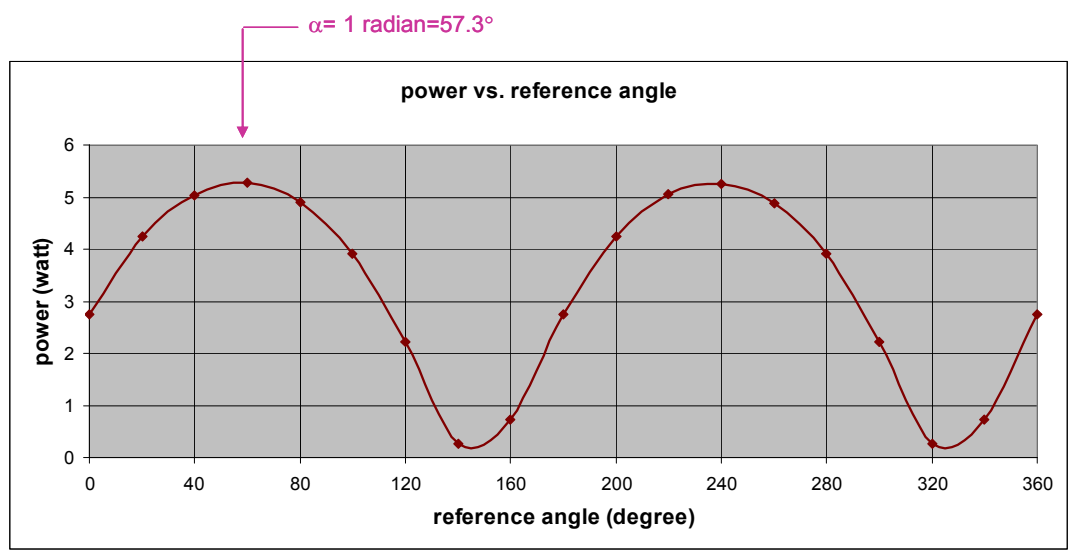

Fig. 4 - a simulation of control reference search

The example assumes that the roll/pitch axis is located at $\phi$ equal to 1.0 radian or $57.3^{\circ}$. The controller searches for this axis by changing slowly the reference angle and recoding the corresponding output power. Figure 4 is a plot of the power versus reference angle. The power is maximal at about $60^{\circ}$ or $240^{\circ}$. This proves the feasibility of searching for the roll/pitch axis without using sensors.

The Matlab/Simulink program for this searching calculation and a sample output are presented in Figure 5. The example has assumed that a CS-WEC with sliding mass of $50 \mathrm{~kg}$ and $1 \mathrm{~m}$ track radius. The wave condition was a swell with $2 \mathrm{~m}$ height at $10 \mathrm{~s}$ period.

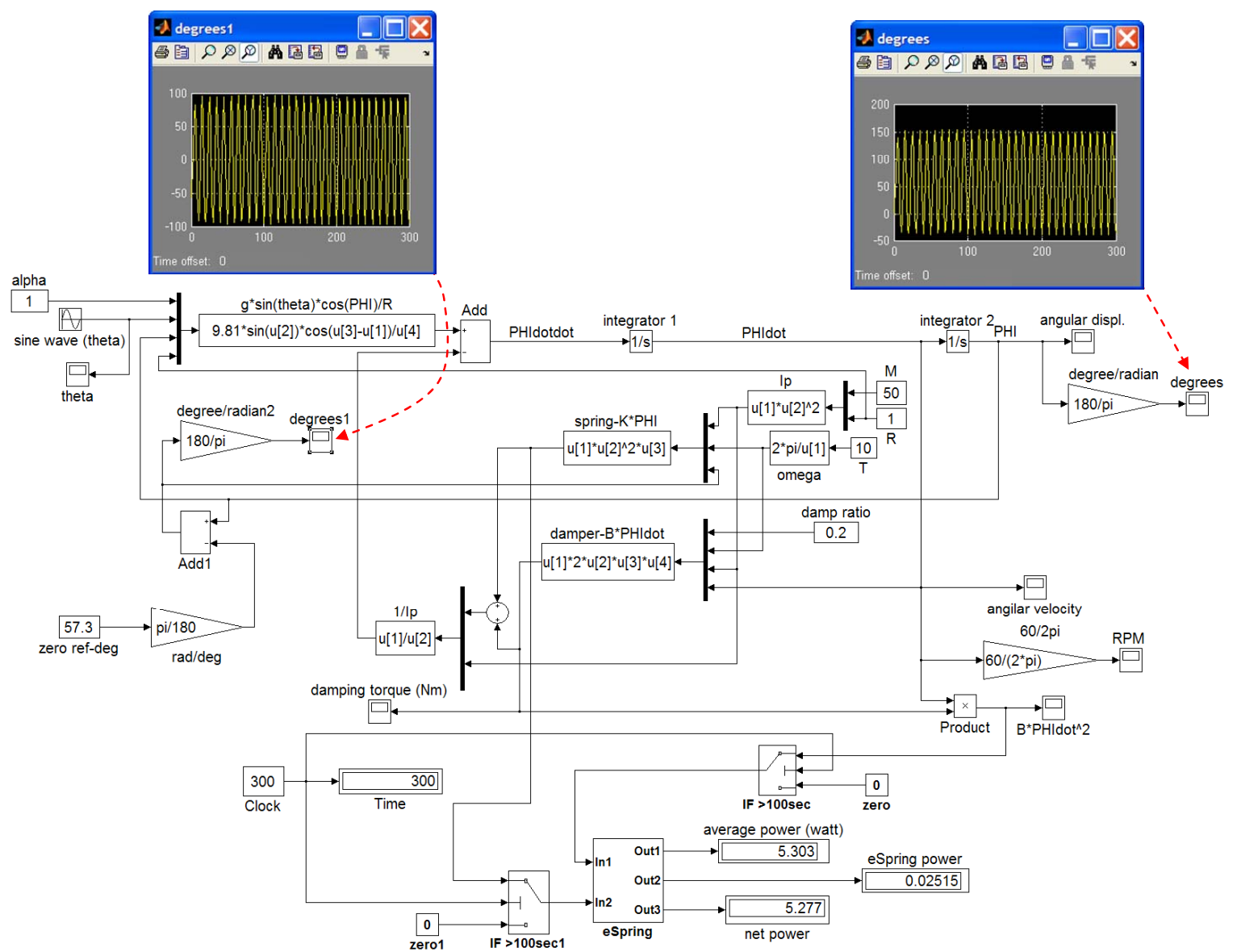

Fig. 5 - Simulink program for demonstrating feedback reference search

The artificial spring (eSpring) consumed very little power and the net harvest was 5.3 watts. The right "scope" shows the mass sliding angle is biased by about $60^{\circ}$ or approximately 1 radian. The 
left scope shows the feedback angle in the range of $\pm 90^{\circ}$ (around the reference) indicating the optimal operation.

\section{Simulation of random waves}

To simulate CS-WEC power harvest in random waves, the wave incline angle $\theta$ in (1) for random wave has to be estimated. Based on a Pierson-Moskowitz wave spectrum [5], random wave heights were computed at different wind speeds. The random waves at each wind speed consist of components in a range of frequencies or equivalent periods. For example, at a wind speed of 15-kt, the wave period content may be approximated by Figure 6. Applying (2), the incline angle amplitude for each period component is calculated. The total incline angle of random wave at a wind speed is calculated by summing these component amplitudes. Since no phase information was available, the phases of the wave components are assumed to be random.

Note that the components at the digitizing periods at 0.5, 1.0 and 1.5s are eliminated in Figure 6. These relatively high-frequency components are associated with short wave lengths of $0.39,1.56$, and $3.51 \mathrm{~m}$ (in deep water), respectively. A buoy might have a diameter larger than these wavelengths; its natural mode frequencies might be too low to respond to these relatively highfrequency excitations. In order to avoid the complication of undefined dynamics that are associated with a specific buoy size, it was assumed that the system would not respond to wave period $\leq 1.5$ seconds.

The wave amplitude versus time for the $15-\mathrm{kt}$ case is shown in the upper plot of Figure 7 . It shows a wave height of more than $1 \mathrm{~m}$, which is consistent with the "significant wave height $(1.27 \mathrm{~m})$ " at 15 -kt wind speed. The lower plot of Figure 7 is the random wave surface incline angle at 15-kt wind, and its FFT frequency spectrum is presented in Figure 8 showing all wave frequencies less than $0.7 \mathrm{~Hz}$.
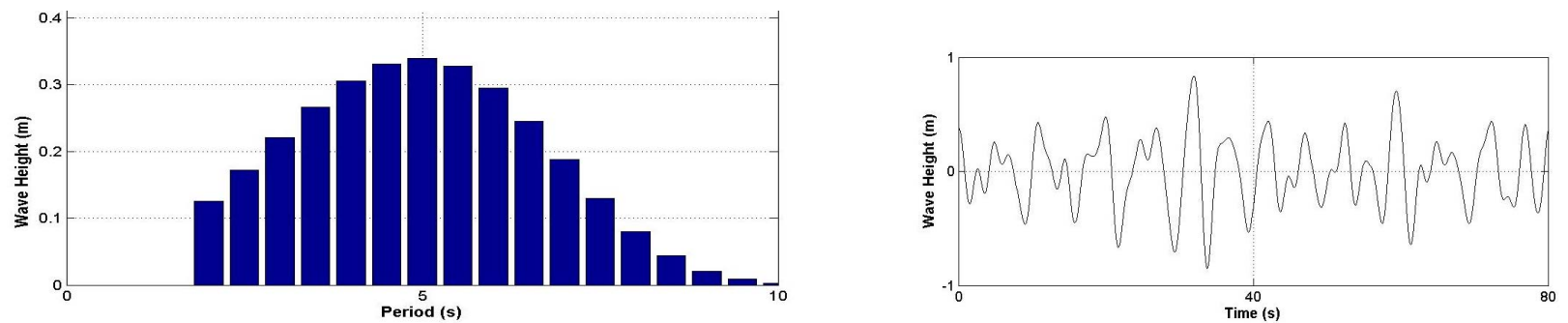

Fig. 6 - 15-kt random wave frequency content with 3 short periods eliminated.
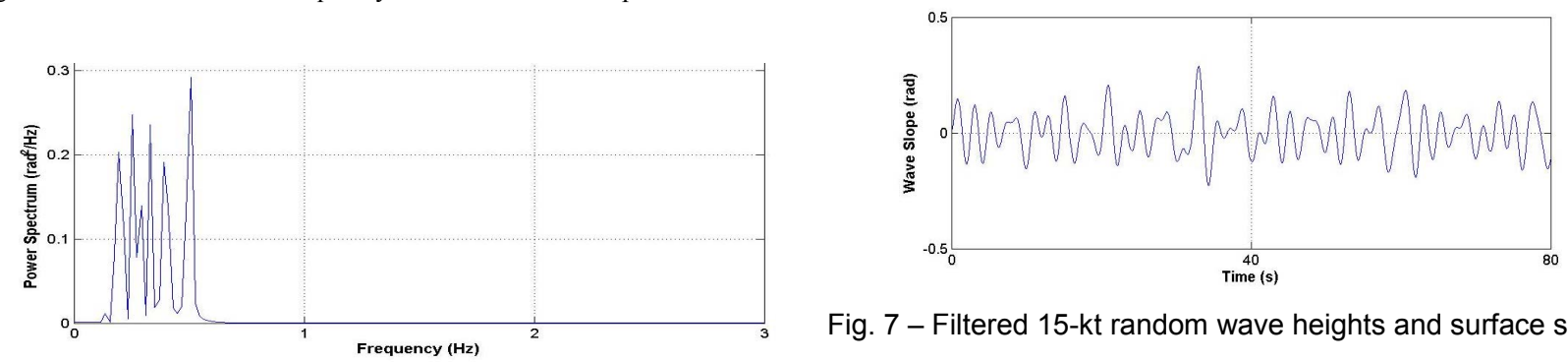

Fig. 7 - Filtered 15-kt random wave heights and surface slopes.

Fig. 8 - Filtered frequency content of random wave surface tilts.

\section{Summary}

The power comparison study between with and without using artificial spring has been concluded for CS-WEC as follows:

The harvested power is always more with the artificial spring than without the spring. With the spring, the power harvest is insensitive to load, and when the waves are low, the advantageous spring effect is more pronounced. 


\section{Acknowledgment}

This paper is supported by 2013 International Corporation Research Fund of Jinan, China, No. 201305057

\section{References}

[1] Cheung, J.T. \& Childress, E.F. III, Ocean Wave Energy Harvesting, DARPA Phase 3 Final Report, Teledyne Scientific \& Imaging, April 2008.

[2]Chen, H.M. and DelBalzo, D.R., "Circular-slide wave energy converter," Proceedings of Ocean Waves Workshop, New Orleans 28 February 2013. (http://scholarworks.uno.edu/oceanwaves/2013).

[3] McCormick, M. E., Ocean Wave Energy Conversion, Dover Publication Inc., 2007, Chapter 2.

[4]SI Ocean report, Ocean Energy: State of the Art, Dec, 2012, pages, 15, 16 and 30 (http://www.emec.org.uk/marine-energy/wave-devices/).

[5] Chen, H.M. and DelBalzo, D.R., "Circular-Slide Wave Energy Converter in Random Waves," presented at Oceans'13, MTS/IEEE San Diego, 23-26 September, 2013. 